\title{
Fractional Flow Reserve Method in Cardiac Catheterization Laboratory without Cardiosurgical Backup: Initial Experiences
}

\author{
Ivan Simic ${ }^{1,2^{*}}$, Vladimir Zdravkovic ${ }^{1,2}$, Rada Vucic ${ }^{1,2}$, Violeta Iric-Cupic ${ }^{1,2}$, Goran Davidovic ${ }^{1,2}$, Vladimir Ignjatovic ${ }^{2}$, Dragic \\ Bankovic $^{3}$ \\ ${ }^{1}$ Faculty of Medical Sciences, University of Kragujevac, Svetozara Markovica 69, Kragujevac, Serbia; ${ }^{2}$ Clinic for Cardiology, \\ Clinical Center Kragujevac, Zmaj Jovina 30, Kragujevac, Serbia; ${ }^{3}$ Department of Mathematics and Informatics, Faculty of \\ Science, University of Kragujevac, Radoja Domanovica 12, Kragujevac, Serbia
}

\begin{abstract}
Citation: Simic I, Zdravkovic V, Vucic R, IricCupic V, Davidovic G, Ignjatovic V, Bankovic D. Fractional Flow Reserve Method in Cardiac Catheterization Laboratory withou Cardiosurgical Backup: Initial Experiences. OA Maced J Med Sci. 2013 Dec 15; 1(1):50-53. http://dx.doi.org/10.3889/oamjms.2013.010

Key words: FFR; intermediate stenosis; PCl; cardiosurgical backup; QCA

"Correspondence: Ivan Simic, MD, PhD. UI. Vase Čarapića 6/22, 34000 Kragujevac, Serbia Tel: $+381641619940 ;+38134505088$; Fax: +381 34 370089. E-mail: ivansimickg@gmail.com

Received: 15-Sep-2013; Revised: 26-Oct2013; Accepted: 27-Oct-2013; Online first: 31-Oct-2013

Copyright: ๑ 2013 Simic I. This is an openaccess article distributed under the terms of the Creative Commons Attribution License, which permits unrestricted use, distribution, and reproduction in any medium, provided the original author and source are credited.

Competing Interests: The authors have declared that no competing interests exist.
\end{abstract}

\begin{abstract}
Background: Coronary artery disease is the most common cause of death in a modern world. This dictates the development a network of Catheterization laboratories without cardiosurgical capabilities.

Aim: We postulate that the most valuable tool in the decision process on myocardial revascularization is fractional flow reserve (FFR), especially when we deal with borderline coronary lesions.

Material and Methods: A total of 72 patients with 94 intermediate coronary stenosis $(30 \%-70 \%$ diameter reduction) were included in this study. We tested FFR and angiography based decision model on myocardial revascularization.

Results: Mean FFR value on left anterior descending coronary artery (LAD) was lower than in others two arteries $(p=0.017)$. FFR after percutaneous coronary intervention $(\mathrm{PCl})$ was significantly better $(p<0.0001)$. The decision for PCl predominates before FFR diagnostics, but after FFR the decision is quite opposite. There is a weak negative correlation between FFR and diameter of stenosis assessed by angiography $(r=-0.245 p=0.038)$ and positive correlation between diameter of stenosis assessed by angiography and by quantitative coronary angiography (QCA) $(r=0.406$ $\mathrm{p}<0.0005)$.
\end{abstract}

Conclusion: Our results strongly suggest that FFR is necessary tool in centers without possibilities of heart team onsite consultation and that prevents numerous unnecessary $\mathrm{PCl}$.

\section{Introduction}

The functional significance of coronary artery stenosis of intermediate severity is important in determining revascularization strategy in patients. By the early 1990s, fractional flow reserve (FFR) emerged as an important physiologic adjunct to coronary angiography for the assessment of intermediate lesions, directing multivessel percutaneous revascularization and guiding stent deployment. Pressure-derived fractional flow reserve (FFR) assessment has been extensively described and validated as a technique capable of identifying functionally significant $[1,2]$.
In centers without cardiosurgical capabilities, lack of cardiosurgeon in heart team, may cause subsequently inadequate decisions on myocardial revascularization, which make the decision process harder.

\section{Materials and Methods}

\section{Study population}

Seventy two (72) patients with 94 intermediate coronary stenosis were examined 
between 2006 - 2009. All patients submitted to coronary angiography, for any reason, were eligible and the decision to use FFR was left entirely at the operator's discretion. Patients were eligible for the study if they had a de novo moderate (30\%-70\% diameter) stenosis in a native coronary arteries with diameter $>2.5 \mathrm{~mm}$. Patients were excluded from the study if they had a history of bronchial asthma or bronchial hyperreactivity, severe renal failure, target vessel with multiple or tandem lesions, previous myocardial infarction in the territory of the target vessel.

Table 1: Baseline characteristics.

\begin{tabular}{ll}
\hline Number of patients & 72 \\
Number of lesions & 94 \\
Number of FFR positive lesions & $26(24.44 \%)$ \\
Overall costs before FFR 94 & $180 €$ \\
Overall costs after FFR 105 & $400 €$ \\
Age (yrs) & $58.6 \pm 8.57$ \\
Male gender & $71 \%$ \\
A-DS (\%) & $53.45 \pm 8.80$ \\
QCA-DS (\%) & $45.40 \pm 8.78$ \\
FFR before PCl & $80.62 \pm 9.89$ \\
FFR after PCI & $89.81 \pm 5.05$ \\
Smoking & $54 \%$ \\
Hypercholesterolemia & $76 \%$ \\
Hypertension & $82 \%$ \\
Obesity & $43 \%$ \\
Family history of CAD & $38 \%$ \\
Diabetes mellitus & $27 \%$ \\
Acetylsalicylic acid & $97 \%$ \\
Clopidogrel & $66 \%$ \\
Beta-blockers & $80 \%$ \\
Calcium antagonists & $51 \%$ \\
Nitrates & $53 \%$ \\
Angiotensin-converting enzyme inhibitor & $78 \%$ \\
Statin & $74 \%$ \\
Diuretics & $32 \%$ \\
\hline
\end{tabular}

Assessment of the target vessel (a major coronary artery or major branch with visual estimated stenosis of $\geq 50$ diameter stenosis, DS\%) reference diameter by angiography and on-line QCA (Siemens Axiom Artis System) was mandatory. Quantitative coronary angiography was performed using the contrast-filled distal guiding catheter for calibration. The angiographic projection with the most severe diameter narrowing without foreshortening was used for analysis of stenosis severity.

\section{FFR measurements}

The ratio of the distal and the aortic pressure on maximal hyperemia, or pressure-derived FFR (FFR $=\mathrm{Pd} / \mathrm{Pa})$, has been extensively validated $[3,4]$. The stenosis was considered to be of physiologic significance only if the FFR was $\leq 0.75$. As per protocol, patients were deferred from coronary intervention if their FFR was $>0.75$.

After a diagnostic coronary angiography, $6 \mathrm{Fr}$ to 7Fr guiding catheter without side holes was used to engage the coronary artery. Heparin was administered intravenously (bolus dose of $100 \mathrm{U} / \mathrm{kg}$ ). A 0.014-inch sensor-tipped Pressure Wire (RADI Medical, Uppsala, Sweden) was set at zero, calibrated, and advanced through the guiding catheter. The transducer was aligned first with the catheter tip to ensure that the pressure wire and coronary catheter recorded the same pressure, and then the transducer was positioned just beyond the stenosis. Mean coronary pressures proximal and distal to the stenosis were measured under maximum coronary hyperemia induced by administration of intracoronary adenosine (140 $\mathrm{mg}$ for the left coronary artery and $60 \mu \mathrm{g}$ for the right coronary artery) after i.c. bolus of nitroglycerine $(200 \mu \mathrm{g}$ for the left and $100 \mu \mathrm{g}$ for the right coronary artery). Measurements were recorded 5 seconds after adenosine bolus.

This was a retrospective, nonrandomized, observational, single-center study.

\section{Statistical considerations}

Values of examined parameters were analyzed using descriptive statistics, as mean and standard deviation. Statistical significance was defined at a $P$ value of $<0.05$. Comparison of two values of the same descriptive was done using paired $\mathrm{T}$ test. Correlation was calculated using Pearson's correlation coefficient. Statistical analyses were performed using Statistical Package for Social Sciences (SPSS) statistical software (Chicago, Illinois, USA).

\section{Results}

In our study population, males were dominant (Table 1). Smoking, hypertension and hypercholesterolemia were most frequent risk factors for coronary artery disease.

Pretreatment with DAPT, beta blockers, ACE inhibitors and statins was consistent with guidelines. Cost of FFR diagnostic is slightly higher than without FFR in our institution. Values of diameter stenosis assessed by angiography and by QCA are in intermediate range. Mean FFR value is at upper limit. FFR value on LAD is lower than in others two arteries. The presumed explanation for these results lies in the fact that FFR is a parameter which depends of stenosis severity and amount of viable distal myocardium, and LAD has greater vascularization area. Stenosis on LAD was dominant (Fig. 1).

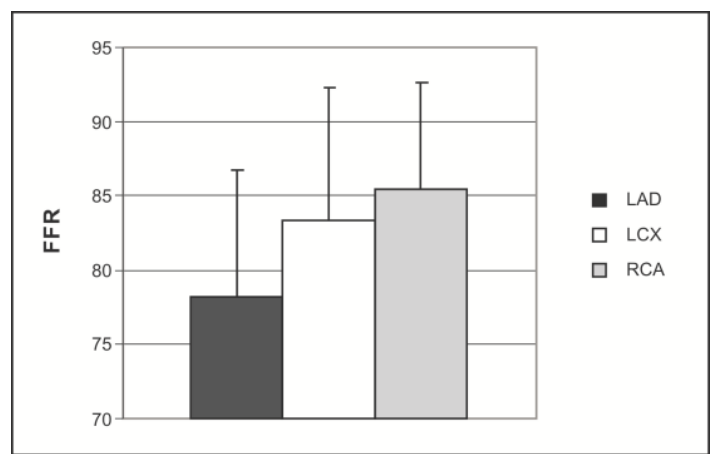

Figure 1: Mean values of FFR on different coronary arteries ( $L A D$ $L C X p=0.031 ; L A D-R C A p=0.017$ ). 
FFR after $\mathrm{PCl}$ is significantly better than before $\mathrm{PCl}$, as we expected (Fig. 2).

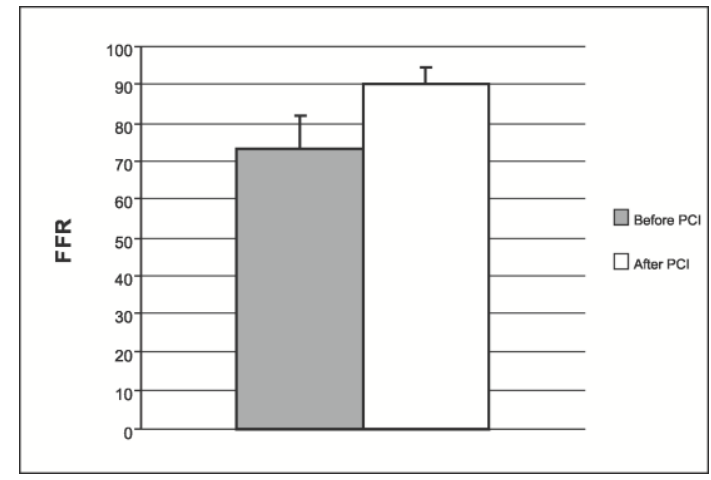

Figure 2: FFR values before and after $\mathrm{PCl}(\mathrm{p}<0.0001)$.

There is a weak negative correlation between FFR and diameter of stenosis assessed by angiography and positive correlation between diameter of stenosis assessed by angiography and by QCA (Table 2).

Table 2: Correlation between different parameters.

\begin{tabular}{lccl}
\hline $\begin{array}{l}\text { Correlated } \\
\text { parameters }\end{array}$ & $\mathbf{R}$ & $\mathbf{p}$ & Comment \\
\hline FFR-A & -0.245 & 0.038 & Weak negative correlation \\
FFR-QCA & -0.072 & 0.148 & No correlation \\
A-QCA & 0.406 & $<0.0005$ & Positive correlation \\
Age-FFR & 0.205 & 0.084 & No correlation \\
\hline
\end{tabular}

There is no correlation between other parameters. The decision to perform $\mathrm{PCl}$ predominates before FFR diagnostics, but after FFR the decision is quite opposite (Fig. 3 ).

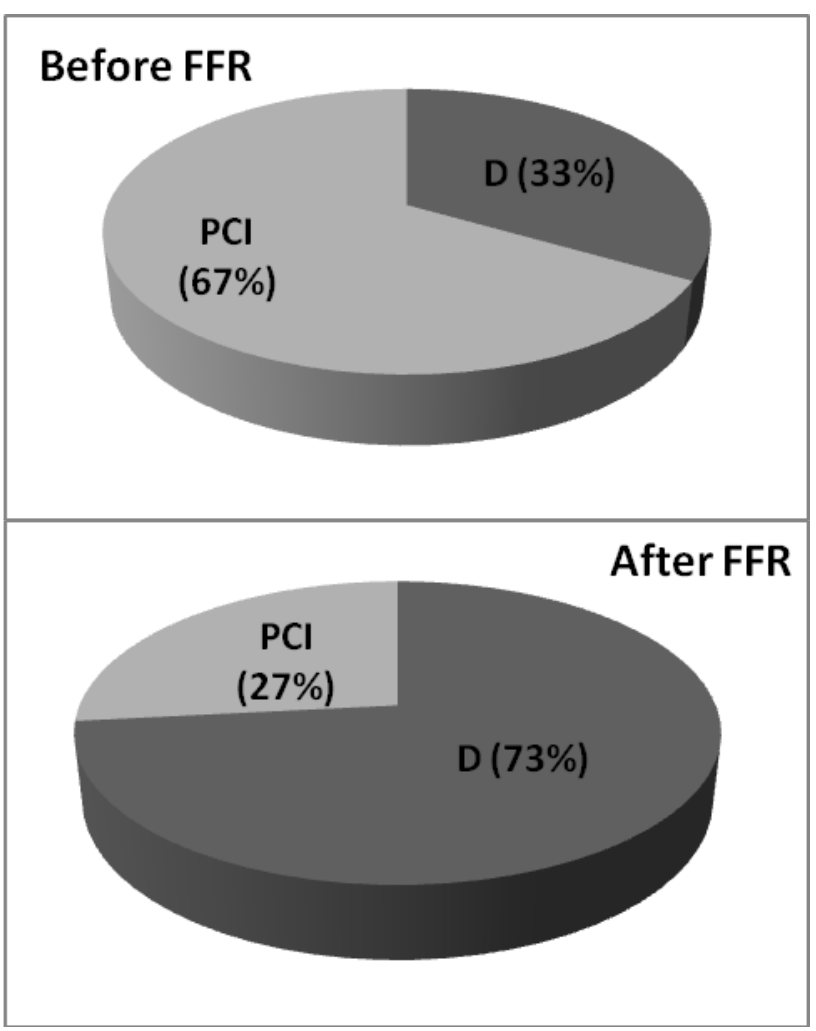

Figure 3: Decision to perform or defer (D) PCI before and after FFR.

\section{Discussion}

Centers without cardiosurgical capabilities use more efforts to indicate right therapeutical strategies for the patients. Among other invasive diagnostic modalities, like IVUS and OCT, FFR is the method that offers best diagnostic accuracy and provides reliable information on functional significance of coronary stenosis. This is of great importance in institution without cardiosurgical backup. Adequate on site diagnosis save time and money, compared to transferring patient or coronary angiogram to other hospital with cardiosurgeon for heart team decision (even then the decision is often based just on eyeballing).

Most patients undergo to catheterization before a noninvasive evaluation [5]. In this setting, determining the appropriate management of an intermediate coronary lesion can be challenging. Measuring FFR has been shown by recent studies to be an accurate method for determining the physiologic significance of an intermediate coronary lesion [6-8]. The lack of benefit of $\mathrm{PCl}$ on non-ischemia producing lesions has been clearly documented [9].

The recommended cut-off value for FFR is $0.75[10,11]$ with a 'grey zone' between 0.75 and 0.80 . In our routine strategy, the choice of a threshold is at operators discretion and location of stenosis. Just minority of examined lesions (24.44\%) had positive FFR and they were treated in the same or staged procedure. Lowest FFR values on LAD indicate inaccuracy of visual assessment of borderline stenosis on this artery due to largest distributional area. Stents were successfully implanted according to local practice in all patients with an implantation pressure of 14-16 atm. The prognostic value of FFR after coronary stent implantation has been proved in a multicentre registry [12].

In our study, FFR values after procedure are little lower then recommended. Possible explanation for these results lies in fact that many of our patients had diffuse atherosclerosis that may cause a graded continuous pressure fall over the whole arterial length [13]. Since we used intracoronary instead intravenous adenosine, we did not assess the FFR in the whole artery (pull back curve). In clinical follow up, we did not have stent thrombosis and restenosis documented by angiography. But, majority of our patients had a preserve EF\% and single vessel disease. This, perhaps, indicate effect of more profound reactive hyperemia after stent deployment that decreases FFR values recorded immediate after $\mathrm{PCl}$. Furthermore, the statistical power of this study may be limited by the single centre approach.

We did not found correlation between age and FFR. There is a weak negative correlation between visual assessment of diameter stenosis and FFR but angiography remains to be unreliable. QCA does not correlate with FFR and cannot be used in decision 
making process in case of borderline lesions. But, we found positive correlation between angiography and QCA evaluation of lesion severity. Explanation is that both methods are based on lumenography and morphology.

In our study, waste majority of stenoses are considered as angiography significant before FFR but final decision after FFR was completely different. So, numerous unnecessary $\mathrm{PCl}$ and surgical team consultations were avoided. Similar results were recently found in the group of patients with NSTEMI [14]. FFR measurement reduced diagnostic variability and changed operators' treatment options in these groups of patients.

Finally, with relatively high price of Pressure Wire in our country, $\mathrm{PCl}$ with DES penetration of about $25 \%$ in our hospital and accompanying therapy after PCI, FFR guided decisions are little more expensive then without FFR. This experience is opposite to those in other countries [15]. Possible explanations of these phenomenons lies in cheap working hours of medical stuff, poor DES penetration and higher prices of Pressure Wire in our country. So, in our local conditions, due to reimbursement problems, we must pay much attention on patient's selection for functional diagnostics of intermediate coronary stenosis. We strongly recommend performance of exercise stress test or dobutamine stress echo test before FFR. But, in our opinion, FFR is the best additional invasive diagnostic tool in CathLabs without cardiosurgical backup, providing most valuable diagnostic information.

\section{References}

1. De Bruyne B, Pijls NH, Paulus WJ, et al. Transstenotic coronary pressure gradient measurement in humans: in vitro and in vivo evaluation of a new pressure monitoring angioplasty guide wire. J Am Coll Cardiol. 1993; 22:119-26.

2. De Bruyne B, Bartunek J, Sys SU, et al. Simultaneous coronary pressure and flow velocity measurements in humans. Feasibility, reproducibility, and hemodynamic dependence of coronary flow velocity reserve, hyperemic flow versus pressure slope index, and fractional flow reserve. Circulation. 1996; 94:1842-49.

3. Pijls NH, Van Gelder B, Van der Voort P, et al. Fractional flow reserve. A useful index to evaluate the influence of an epicardial coronary stenosis on myocardial blood flow. Circulation. 1995; 92:3183- 93.

4. Pijls NH, De Bruyne B, Peels K, et al. Measurement of fractional flow reserve to assess the functional severity of coronary-artery stenoses. N Engl J Med. 1996; 334:1703 -8.

5. Topol EJ, Ellis SG, Cosgrove DM, et al. Analysis of coronary angioplasty practice in the United States with an insuranceclaims data base. Circulation. 1993;87:1489-97.

6. De Bruyne B, Bartunek J, Sys SU et al. Relation between myocardial fractional flow reserve calculated from coronary pressure measurements and exercise-induced myocardial ischemia. Circulation. 1995; 92:39-46.

7. Simic I, Zdravkovic V, Vucic R, et al. Fractional flow reserve in patients with intermediate values of Duke treadmill score and borderline coronary lesions. Arch Biol Sci. 2013;65:1285-92.

8. Fearon WF, Takagi A, Jeremias $A$, et al. Use of fractional myocardial flow reserve to assess the functional significance of intermediate coronary stenoses. Am J Cardiol. 2000; 86:10134.
9. Pijls $\mathrm{NH}$, van Schaardenburgh $\mathrm{P}$, Manoharan $\mathrm{G}$, et al. Percutaneous coronary intervention of functionally nonsignificant stenosis: 5-year follow-up of the DEFER Study. J Am Coll Cardiol. 2007;49:2105-11.

10. Heyndrickx GR and Wijns W. Coronary flow reserve calculated from pressure measurements in humans. Validation with positron emission tomography. Circulation. 1994; 89:1013-22.

11. Pijls N, van Son J, Kirkeeide R, De Bruyne B and Gould K. Experimental basis of determining maximum coronary, myocardial, and collateral blood flow by pressure measurements for assessing functional stenosis severity before and after percutaneous transluminal coronary angioplasty. Circulation. 1993;87:1354-67.

12. Pijls $\mathrm{NH}$, Klauss $\mathrm{V}$, Siebert $\mathrm{U}$, et al. Coronary pressure measurement after stenting predicts adverse events at followup. Circulation. 2002; 105:2950-4.

13. De Bruyne B, Hersbach F, Pijls NH, et al. Abnormal epicardial coronary resistance in patients with diffuse atherosclerosis but "normal" coronary angiography. Circulation. 2001;104:2401-6.

14. Carrick D, Behan M, Foo F, et al. Usefulness of fractional flow reserve to improve diagnostic efficiency in patients with nonST elevation myocardial infarction. Am J Cardiol. 2013; 111:45-50

15. Fearon WF, Yeung AC, Lee DP, Yock PG and Heidenreich PA. Cost-effectiveness of measuring fractional flow reserve to guide coronary interventions. Am Heart J. 2003;145:882-7. 